\title{
SuperTML: Two-Dimensional Word Embedding for the Precognition on Structured Tabular Data
}

\author{
Baohua Sun, Lin Yang, Wenhan Zhang, Michael Lin, Patrick Dong, Charles Young, Jason Dong \\ Gyrfalcon Technology Inc. \\ 1900 McCarthy Blvd. Milpitas, CA 95035 \\ \{baohua.sun, lin.yang, wenhan.zhang\}@gyrfalcontech.com
}

\begin{abstract}
Tabular data is the most commonly used form of data in industry according to a Kaggle ML and DS Survey. Gradient Boosting Trees, Support Vector Machine, Random Forest, and Logistic Regression are typically used for classification tasks on tabular data. DNN models using categorical embeddings are also applied in this task, but all attempts thus far have used one-dimensional embeddings. The recent work of Super Characters method using twodimensional word embeddings achieved state-of-the-art results in text classification tasks, showcasing the promise of this new approach. In this paper, we propose the SuperTML method, which borrows the idea of Super Characters method and two-dimensional embeddings to address the problem of classification on tabular data. For each input of tabular data, the features are first projected into twodimensional embeddings like an image, and then this image is fed into fine-tuned two-dimensional CNN models for classification. The proposed SuperTML method handles the categorical data and missing values in tabular data automatically, without any need to pre-process into numerical values. Comparisons of model performance are conducted on one of the largest and most active competitions on the Kaggle platform, as well as on the top three most popular data sets in the UCI Machine Learning Repository. Experimental results have shown that the proposed SuperTML method have achieved state-of-the-art results on both large and small datasets.
\end{abstract}

\section{Introduction}

In data science, data is categorized into structured data and unstructured data. Structured data is also known as tabular data, and the terms will be used interchangeably. Anthony Goldbloom, the founder and CEO of Kaggle observed that winning techniques have been divided by whether the data was structured or unstructured [37]. Currently, DNN models are widely applied for usage on unstructured data such as image, speech, and text. According to Anthony, "When the data is unstructured, its definitely CNNs and RNNs that are carrying the day" [37]. The successful CNN model in the ImageNet competition [29] has outperformed human for image classification task by ResNet [16] since 2015. And the following efforts of PolyNet [39], SEnet [17], and NASNet [40] keep breaking the records. Current state of the art on ImageNet given by PNASNe [22] achieves $82.9 \%$ Top1 accuracy.

On the other side of the spectrum, machine learning models such as Support Vector Machine (SVM), Gradient Boosting Trees (GBT), Random Forest, and Logistic Regression, have been used to process structured data. According to a recent survey of 14,000 data scientists by Kaggle (2017), a subdivision of structured data known as relational data is reported as the most popular type of data in industry, with at least $65 \%$ working daily with relational data. Regarding structured data competitions, Anthony says that currently XGBoost is winning practically every competition in the structured data category [13]. XGBoost [5] is one popular package implementing the Gradient Boosting method. Other implementations include lightgbm [19], and catboost [27].

Recent research has tried using one-dimensional embedding and implementing RNNs or one-dimensional CNNs to address the TML (Tabular data Machine Learning) tasks, or tasks that deal with structured data processing [21, 35], and also categorical embedding for tabular data with categorical features [15, 7]. One-dimensional embeddings such as word2vec [23], GLoVe [25], fastText [18], ELMO [26], BERT [9], and Open AI GPT [28] are widely used in NLP tasks, and as such data scientists have tried to adapt them to TML tasks. These one-dimensional embeddings project each token into a vector containing numerical values. For example, a word2vec embedding [23] could be a one-dimensional vector that acts like a 300 by 1 array.

However, this reliance upon one-dimensional embeddings may soon come to change. Recent NLP research 


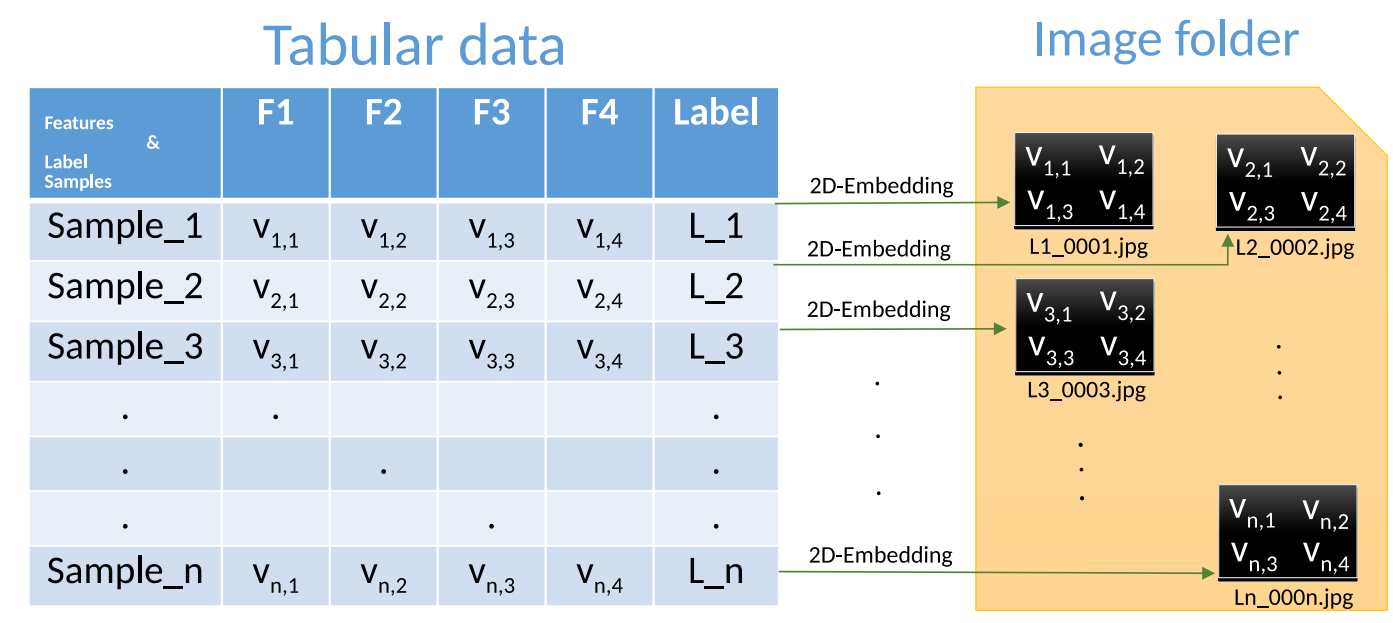

Figure 1: An example of converting training data from tabular into images with two-dimensional embeddings of the features in the tabular data. Therefore, the problem of machine learning for tabular data is converted into an image classification problem. The later problem can use pretrained two-dimensional CNN models on ImageNet for finetuning, for example, ResNet, SE-net and PolyNet. The tabular data given in this example has $\mathrm{n}$ samples, with each sample having four feature columns, and one label column. For example, assume the tabular data is to predict whether tomorrow's weather is "Sunny" or "Rainy". The four features F1, F2, F3, and F4 are respectively "color of the sky", "Fahrenheit temperature", "humidity percentage", and "wind speed in miles per hour". Sample_1 has class label L1="Sunny", with four features values given by $v_{1,1}=$ "blue", $v_{1,2}=55, v_{1,3}=$ "missing", and $v_{1,4}=17$. The two-dimensional embedding of Sample_1 will result in an image of "Sunny_0001.jpg" in the image folder. The four feature values are embedded into the image on different locations of the image. For example, $v_{1,1}$ is a categorical value of color "blue", so the top left of the image will have exactly the alphabets of "blue" written on it. For another example, $v_{1,2}$ is a numerical value of " 23 ", so the top right of the image will have exactly the digits of " 23 " written on it. For yet another example, $v_{1,3}$ should be a numerical value but it is missing in this example, so the bottom left of the image will have exactly the alphabets of "missing" written on it. Other ways of writing the tabular features into image are also possible. For example, "blue" can be written in short as a single letter "b" if it is distinctive to other possible values in its feature column. The image names will be parsed into different classes for image classification. For example, L1 = L2 = "Sunny", and L3 = Ln ="Rainy". These will be used as class labels for training in the second step of SuperTML method.

has shown that the two-dimensional embedding of the Super Characters method [33] is capable of achieving stateof-the-art results on large dataset benchmarks. The Super Characters method is a two-step method that was initially designed for text classification problems. In the first step, the characters of the input text are drawn onto a blank image, so that an image of the text is generated with each of its characters embedded as the pixel values in the twodimmensional space, i.e. a matrix. The resulting image is called a Super Characters image. In the second step, Super Characters images are fed into two-dimensional CNN models for classification. The two-dimensional CNN models are trained by fine-tuning from pretrained models on large image dataset, e.g. ImageNet. This process is also known as Transfer Learning [30, 10, 38, 24].

In this paper, we propose the SuperTML method, which borrows the concept of the Super Characters method to address TML problems. For each input, tabular features are first projected onto a two-dimensional embedding and fed into fine-tuned two-dimensional CNN models for classification. The proposed SuperTML method handles the categorical type and missing values in tabular data automatically, without need for explicit conversion into numerical type values.

Experimental results show that this proposed SuperTML method performs well on both large and small datasets. In one instance of the Higgs Boson Machine Learning Challenge dataset, which is "one of the largest and most active competitions on the Kaggle platform" [6], a single model that applied SuperTML manages to analyze 250,000 training instances and 550,000 testing instances and obtain an AMS score of 3.979, a state-of-the-art result that beat the previous best of 3.806 [2]. When using the top three popular databases (ranked by number of times accessed since 2007) from UCI Machine Learning Repository (includes the Iris dataset (150 data instances), Adult dataset (48,482 data in- 
stances), and Wine dataset (178 data instances)), the SuperTML method still achieved state-of-the-art results for all datasets despite this variation in dataset size.

\section{The Proposed SuperTML Method}

The SuperTML method is motivated by the analogy between TML problems and text classification tasks. For any sample given in tabular form, if its features are treated like stringified tokens of data, then each sample can be represented as a concatenation of tokenized features. By applying this paradigm of a tabular sample, the existing CNN models used in Super Characters method could be extended to be applicable to TML problems.

As mentioned in the introduction, the combination of two-dimensional embedding (a core competency of the Super Characters methodology) and pre-trained CNN models has achieved state-of-the-art results on text classification tasks. However, unlike the text classification problems studied in [33], tabular data has features in separate dimensions. Hence, generated images of tabular data should reserve some gap between features in different dimensions in order to guarantee that features will not overlap in the generated image.

SuperTML is composed of two steps, the first of which is two-dimensional embedding. This step projects features in the tabular data onto the generated images, which will be called the SuperTML images in this paper. The conversion of tabular training data to SuperTML image is illustrated in Figure 1, where a collection of samples containing four tabular features is being sorted.

The second step is using pretrained CNN models to finetune on the generated SuperTML images.

Figure 1 only shows the generation of SuperTML images for the training data. It should be noted that for inference, each instance of testing data goes through the same pre-processing to generate a SuperTML image (all of which use the same configuration of two-dimensional embedding) before getting fed into the $\mathrm{CNN}$ classification model.

Considering that features may have different importance for the classification task, it would be prudent to allocate larger spaces for important features and increase the font size of the corresponding feature values. This method, known as SuperTML_VF, is described in Algorithm1.

To make the SuperTML more autonomous and remove the dependency on feature importance calculation done in Algorithm 1 the SuperTML_EF method is introduced in Algorithm 2, It allocates the same size to every feature, and thus tabular data can be directly embedded into SuperTML images without the need for calculating feature importance. This algorithm shows even better results than 1, which will be described more in depth later in the experimental section.
Algorithm 1 SuperTML_VF: SuperTML method with Variant Font size for embedding.

Input: Tabular data training set

Parameter: Image size of the generated SuperTML images Output: Finetuned CNN model

1: Calculate the feature importance in the given tabular data provided by other machine learning methods.

2: Design the location and font size of each feature in order to occupy the image size as much as possible. Make sure no overlapping among features.

3: for each sample in the tabular data do

4: for each feature of the sample do

5: Draw feature in the designated location and font size.

6: end for

7: end for

8: Finetune the pretrained CNN model on ImageNet with the generated SuperTML images.

9: return the trained CNN model on the tabular data

Algorithm 2 SuperTML_EF: SuperTML method with Equal Font size for embedding.

Input: Tabular data training set

Parameter: Image size of the generated SuperTML images Output: Finetuned CNN model

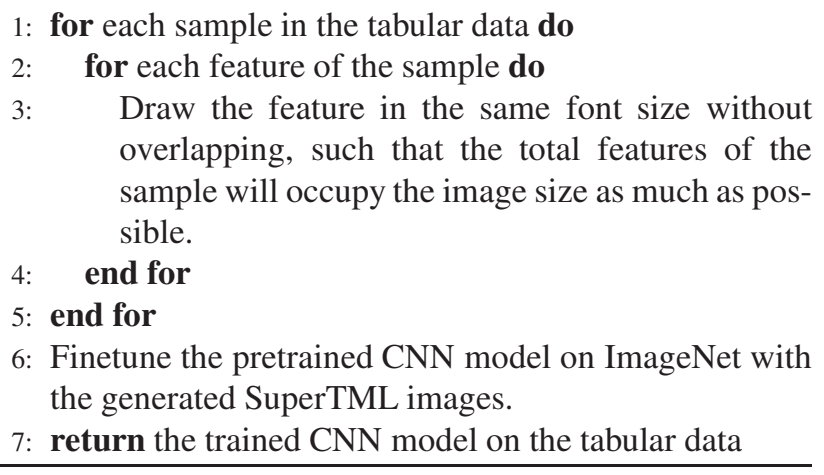

\section{Experiments}

In the experiments described below, we used the top three most popular datasets from the UCI Machine Learning Repository [11] and one well-known dataset from the Kaggle platform. These four datasets cover a variety of TML tasks.

As of the date this paper is written, the Iris dataset [12] is ranked by the UCI Machine Learning Repository as the most popular dataset with $2.41+$ million hits, followed by the Adult dataset [20] (also known as Census Salary dataset) with 1.40+ million hits and the Wine dataset [14] with 1.07+ million hits. Table 1 shows the statistics of these three 
datasets. The data types of the features covers a variety of integer, categorical, real, and missing values.

The Kaggle dataset of Higgs Boson Machine Learning Challenge is also used in the experiments. It "attracted an unprecedented number of participants over a short period of time (May 12, 2014 to Sept 15, 2014)" [2]. "There were in total 1,785 teams participating in the competition, one of the largest and most active ones on the platform website www.kaggle.com" [6].

For the second step in the SuperTML method, the ImageNet pretrained CNN models were used in the experiments. However, a limited amount of pretrained models are publicly available for different size of input. For example, the SE-net published pretrained model only accepts 224x224 input size using Caffe framework; while the PolyNet published model only processes inputs with size $331 \times 331$. In order to mitigate the accuracy difference brought on by usage of different frameworks, NASnet and PNASnet are not used because their Caffe models are not publicly available.

For all the three datasets from the UCI Machine Learning Repository, SuperTML images of size 224×224 are generated. The pre-trained SE-net-154 model was fine-tuned on these three datasets. We also implemented XGBoost and fine-tuned the hyper-parameters on each of the three datasets. For Higgs Boson dataset, SuperTML both images of sizes $224 \times 224$ and $331 \times 331$ were generated for comparison of different pretrained models of SE-net-154 and PolyNet. These two pretrained models have similar performance when working on on ImageNet $(81.32 \%$ forSEnet-154, and $81.29 \%$ for PolyNet) but different input sizes (224x224 for SE-net-154, and 331x331 for PolyNet).

\subsection{Experiments on the Iris dataset}

"This is perhaps the best known database to be found in the pattern recognition literature" [12]. The Iris dataset is widely used in machine learning courses and tutorials. It contains a total of 150 data samples, each of which represents a different subspecies of the Iris genus of flowers (e.g. Iris Setosa, Iris Versicolor, and Iris Virginica). Each sample has a set of four attributes and four corresponding feature values, indicating the measurements of sepal length, sepal width, petal length, and petal width as measured in centimeters.

When implementing SuperTML to this dataset, we came across a multitude of challenges. First, the Iris dataset is very small, with only 150 samples. If we split the training and testing 80:20, it means only there are only 120 training samples and only 40 testing samples for each class. Deep learning models are data hungry, and the CNN models in computer visions are especially well-known for requiring large amounts of labeled images. For example, the ImageNet dataset has over one million images spread relatively

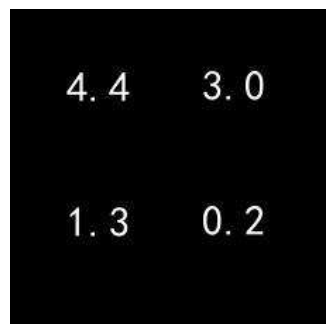

(a) SuperTML_EF image example for Iris data. Each feature is given equal importance in this example.

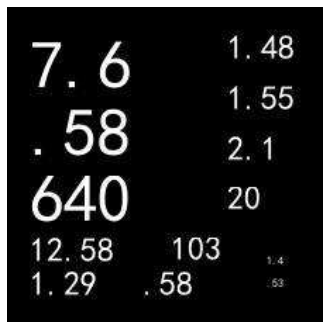

(b) SuperTML_VF image example for Wine data. Features are given different sizes according to their importance.
Figure 2: Examples of generated SuperTML image for Iris and Wine dataset.

equally over one thousand classes. By fine-tuning on this small dataset, there is a high tendency of overfitting. Furthermore, for this Iris dataset, the data types are all real numbers. For methods such as Logistic Regressions, GBT, SVM, and Random Forests, the numerical feature inputs are directly applied to the linear or non-linear models to classify the subspecies. The CNN models used in the SuperTML method must first learn the shapes of these numerical values and then apply nonlinear functions on the extracted image features to classify the Iris subspecies. Just to recognize the shapes of the digits requires quite a lot of data, as shown in MNIST dataset [8].

Figure 2a shows an example of a generated SuperTML image, created using Iris data. Different from existing methods that first convert every feature value into numerical type, as typically done for preprocessing when using XGboost, SVM and etc., the idea of converting tabular features of any data type into string type may seem to increase the difficulty for training model and inference. However, this proposed counter-intuitive method of SuperTML outperforms existing methods as shown in the experimental results. The experimental results of using SE-net-154 shown in Table 2 is based on an 80:20 split of the 150 samples. It shows that the proposed SuperTML method achieves the same accuracy as XGBoost on this small dataset.

\subsection{Experiments on the Wine dataset}

The Wine dataset shares a few similarities to the Iris dataset, so we conducted this experiment immediately after the Iris experiment. This dataset has the similar task of classifying the input samples into one of the three classes and comprises of only 178 samples, making it also a small dataset. The input features are measurements on alcohol, hue, ash, and etc.. In addition, there is no given split of training and testing datasets in this Wine dataset, another similarity between it and the Iris dataset. The number of at- 


\begin{tabular}{|l|r|r|r|r|r|r|r|}
\hline Dataset & Classes & \#Attributes & Train & Test & Total & Data Types & Missing \\
\hline Iris & 3 & 4 & NA & NA & 150 & Real & No \\
Wine & 3 & 13 & NA & NA & 178 & Integer\& Real & No \\
Adult & 2 & 14 & 32,561 & 16,281 & 48,842 & Integer \& Categorical & Yes \\
\hline
\end{tabular}

Table 1: Datasets statistics used in this paper from UCI Machine Learning Repository. The "Missing" in the table indicates whether there are missing values in the data set. The "NA" in the table denotes that there is no given split for the training and testing dataset.

\begin{tabular}{|l|r|r|r|}
\hline Accuracy & Iris(\%) & Wine(\%) & Adult(\%) \\
\hline xgboost & 93.33 & 96.88 & 87.32 \\
GB [4] & - & - & 86.20 \\
\hline SuperTML & $\mathbf{9 3 . 3 3}$ & $\mathbf{9 7 . 3 0}$ & $\mathbf{8 7 . 6 4}$ \\
\hline
\end{tabular}

Table 2: Model accuracy comparison on the tabular data from UCI Machine Learning Repository. The splits on Iris and Wine data is $80 \%: 20 \%$ as described in the experimental setup.

tributes is 13 , which is more than 4 times of that of the Iris dataset. In this set, the features data types includes not just real numbers, but also integers. These differences make the classification on Wine data with SuperTML method even harder than in the Iris dataset for the SuperTML image because of the increased number of features and variation in space due to different data types.

For this dataset, we use SuperTML VF, which gives features different sizes on the SupterTML image according to their importance score. The feature importance score is obtained using the XGBoost package [5]. One example of a SuperTML image created using data from this dataset is shown in Figure $2 \mathrm{~b}$. The importance score shows that the feature of color intensity is the most important, so we allocate font size of 48 to it in the $224 \times 224$ image (can be seen in the top right corner). The following features of importance are flavanoids and proline, which were allocated space on the left and given font size 48. This pattern of importance and font size is applied to all features, all the way down to the least important features of proanthocyanins and nonflavanoid phenols, which were placed in the bottom right corner and given a font size of 8 . The results in Table 2 shows that the SuperTML method obtained a slightly better accuracy than XGBoost on this dataset.

\subsection{Experiments on the Adult dataset}

The task of this Adult dataset is to predict whether a persons income is larger or smaller than 50,000 dollars per year based on a collection of surveyed data. Each sample of data (each person) has 14 attributes, including age, gender, education, and etc.. These attributes are stored using a combination of integer, real numbers, and categorical data. It has 32,561 training samples and 16,281 testing samples. Compared with the other two datasets from the UCI Machine
Learning Repository, this relatively large dataset is in favor of deep learning models that implement the SuperTML method.

For categorical features that are represented by strings, the Squared English Word (SEW) method [32] is used. The benefits of using the English word in this format is twofolded. Firstly, the Super Characters method has shown state-of-the-art performance when processing Asian languages, which has their characters written in the form of glyphs enclosed in a squared space. Building of Super Characters, SEW converts each English word into a square comprised of its characters and guarantees that each word will be written in a unique way. Secondly, writing the features expressed by English strings in this format guarantees that each feature occupies the same position without any change caused by the length of the feature string. One example of a generated SuperTML image is given in Figure 3 Table 2 shows the results on Adult dataset. On this dataset, Biau and et. a.1. [4] proposed an Accelerated Gradient Boosting (AGB) model and compared the performance with an original Gradient Boosting (GB) model using a series of fine-tuned hyper-parameters. The best accuracy is given by the GB model when the shrinkage parameter is set at 0.1 . We also tried implementing XGBoost on thisdataset and preprocessed the categorical data by using integer encoding (using the Python pandas library with astype('category')).The XGBoosts best result was $87.32 \%$ accuracy after fine-tuning the number of trees at 48 . We can see that on this dataset, the SuperTML method still has a higher accuracy than the fine-tuned XGBoost model, outperforming it by $0.32 \%$ points of accuracy. 


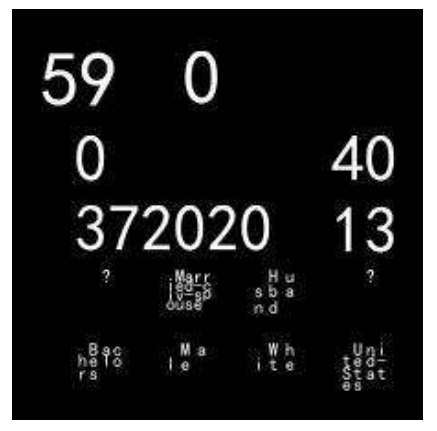

Figure 3: SuperTML image example from Adult dataset. This sample has yearly salary larger than 50k with its features given different sizes in the SuperTML image according to their importance given by third party softwares. This sample has age $=59$, capital gain $=0$, capital loss $=0$, hours per week $=40$, fnlweight $=372020$, education number $=$ 13 , occupation = "?" (missing value as given in the data, it can also be replaced by "MissinngValue" in the SuperTML image), marital status = "Married-civ-spouse", relationship = "Husband", workclass = "?" (missing value $)$, education = "Bachelors", sex = "Male", race = "White", native country = "United-States".

\subsection{Experiments on the Higgs Boson Machine Learning Challenge dataset}

The Higgs Boson Machine Learning Challenge involved a binary classification task to classify quantum events as signal or background. It was hosted by Kaggle, and though the contest is over, the challenge data is available on opendata [2]. It has 25,000 training samples, and 55,000 testing samples. Each example has 30 features, each of which is stored as a real number value. In this challenge, AMS score [1] is used as the performance metric.

The reason why we selected this dataset is two-fold. First, it is a well-known dataset and successful models such as XGBoost and Regularized Greedy Forest have been used in this dataset. Second, the performance metric used in this dataset is AMS score instead of accuracy. It is useful to test the performance of SuperTML method using a different metric and compare its results to other leading options.

The SuperTML images of size $224 \times 224$ are generated for fine-tuning the SE-net models, and the images of size $331 \times 331$ are generated for fine-tuning the PolyNet models. Figure 4a shows an example of example of a background event, which is generated into an SuperTML image of 224x224 through a SuperTML_EF method. Figure 4b shows an example of a signal event, generated through a SuperTML_VF method, which also in an 224x224 image. The $331 \times 331$ SuperTML images are similar to 224x224 images except that each features font size and allocated space

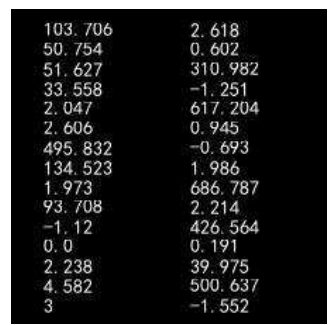

(a) SuperTML image example for Higgs Boson data. Each feature is given equal importance in this example.

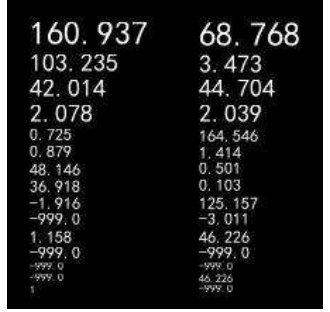

(b) SuperTML image example for Higgs Boson data. Features are given different sizes according to their importance.
Figure 4: Examples of generated SuperTML image for Higgs Boson dataset.

\begin{tabular}{|l|l|}
\hline Methods & AMS \\
\hline DNN by Gabor Meli & 3.806 \\
RGF and meta ensemble & 3.789 \\
Ensemble of neural networks & 3.787 \\
XGBoost & 3.761 \\
\hline SuperTML_EF(224x224) & $\mathbf{3 . 9 7 9}$ \\
SuperTML_VF $(224 \times 224)$ & 3.838 \\
SuperTML_EF $(331 \times 331)$ & 3.934 \\
SuperTML_VF $(331 \times 331)$ & 3.812 \\
\hline
\end{tabular}

Table 3: Comparison of AMS score on Higgs Boson dataset for different methods. The first four rows are top rankers in the leaderboard in the Higgs Boson Challenge.

is increased.

As pointed out by [5], the AMS is an unstable measure, and AMS was not chosen as a direct objective for XGBoost implementation in this challenge. For simplicity, crossentropy loss is still used in this dataset as an objective to minimize. Table 3 shows the comparison of different algorithms. The DNN method and neural networks used in the first and third rows are using the numerical values of the features as input to the models, which is different from the SuperTML method of using two-dimensional embeddings. It shows that SuperTML_EF method gives the best AMS score of 3.979. The PolyNet models trained with larger size of $331 \times 331$ does not help improve the AMS score. In addition, the SuperTML_EF gives better results than SuperTME_VF results for both $224 \times 224$ and $331 \times 331$ image sizes, which indicates SuperTML method can work well without the calculation of the importance scores.

\subsection{Error Analysis}

The experiment using the SuperTML method on the Iris dataset with 80:20 split for training and testing had 2 in- 


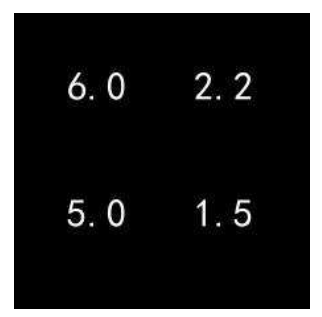

(a) The test SuperTML image of a virginica sample.

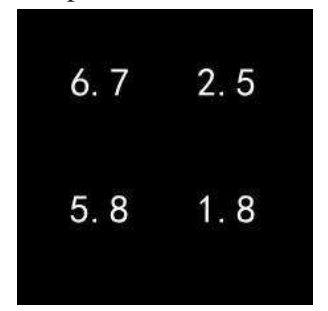

(c) First example from training set with label "virginica".

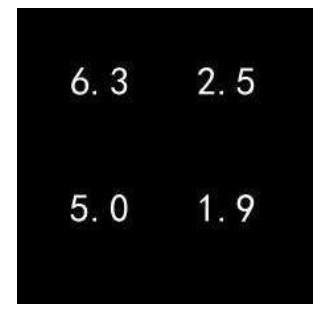

(e) Third example from "virginica". training set with label

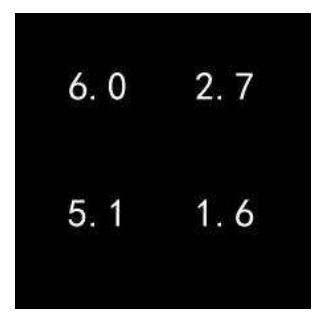

(b) One example from training set with label "versicolor".

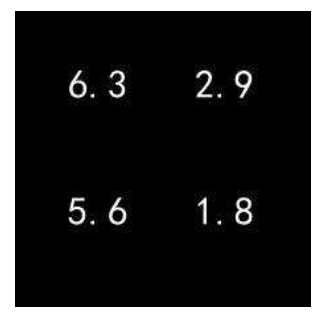

(d) Second example from training set with label "virginica".

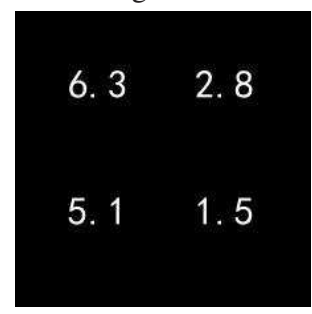

(f) Fourth example from training set with label "virginica".
Figure 5: Error analysis on an Iris-viginica input wrongly predicted as Iris-versicolor. These six samples have the common integer for each of the four feature values, namingly $6,2,5$, and 1 . But these samples may have different decimal values. Figure $5 \mathrm{c}$-Figure $5 \mathrm{f}$ are the only four training samples with the integer portion of the feature values same as Figure 5 a But one "versicolor" sample from training set as shown in Figure 5b not only has the same integer part as Figure 5a but also has more similar shapes of decimal part for each of the four feature values to Figure 5a than the other four samples. The $\mathrm{CNN}$ models that learn the classification model based on the shape of the feature values written on the image have high tendency to classify the example in Figure $5 \mathrm{a}$ as the same category of Figure $5 \mathrm{~b}$.

correct predictions. We will be taking one of the wrongly predicted sample in the testing dataset for error analysis. Its ground truth label is Iris-virginica, but was incorrectly classified as Iris-versicolor. Its four features are 6.0, 2.2, 5.0, and 1.5 respectively, as shown in Figure 5a All the training samples with common integer portion for each of the four feature values, namely $6,2,5$, and 1 are taken into comparison in Figure 5b-Figure 5f. However, these samples may have different decimal values. It shows that this SuperTML image of this virginica example in Figure 5allooks more like the versicolor sample in Figure Figure 5b than the other virginica samples in Figure 5c-Figure 5f] when the shape of numbers in decimal portion is compared. Hence, the testing sample of virginica in Figure 5a is more likely to be classified as versicolor, which is the label for Figure $5 \mathrm{~b}$

The features in this Iris dataset are all numerical values without missing numbers. During model training, these SuperTML images of numerical features are fed into the twodimensional CNN model which classifies images based on the pixel values and the relationship between pixels. At inference time, the model classifies the samples based on the appearance of the features in real-valued numbers. However, the numerical values have some hidden relationship behind the shape of the digits, such as 6.01 and 5.999. They both approximate to the number 6.00 even though their shape is not alike. This is hard for the CNN model to learn.

\section{Conclusion and Future Work}

The proposed SuperTML method borrows the idea from Super Characters and two-dimensional embedding and finetunes the pre-trained CNN models on unstructured data for transfer learning the structured data in the tabular form. Experimental results shows that the proposed SuperTML method has achieved state-of-the-art results on both large and small tabular dataset. As low power domain specific CNN accelerators [34, 31] become available in the market, the SuperTML method can realize its huge potential for practical applications in the real world. For example, for IoT (Internet of Things) applications in smart homes, current machine learning solutions implemented at the edge level are still using Logistic Regression models. These regression models are computationally inexpensive but are expected to be much less accurate when compared to large models like CNN. Using these low-power CNN accelerators with the SuperTML method, it will become possible to provide low-power and high accuracy models at the edge devices. The future work is projected to go in four directions. First, given the success of Super Characters method [33, 32] in text classification, categorical type of data, and also missing value calculations, the SuperTML method should be able to be directly supplement or even replace the current models in that field. Unlike numerical features, the categorical feature has all the information written in the text. Second, compared with not only the Gradient Boosting method, but also the one-dimensional embedding based RNN and CNN methods, SuperTML could become the new state-ofthe-art in computing and solving TML tasks. Third, the SuperTML method could be enlarged to provide support for 
more powerful CNNs such as NASNet, PNASnet, and the others. Fourth, by modifying and improving the model architectures, variant feature importance calculations could be improved in order to find a more accurate way to implement attention [3, 36] scheme.

\section{References}

[1] Claire Adam-Bourdarios, Glen Cowan, Cecile Germain, Isabelle Guyon, Balazs Kegl, and David Rousseau. Learning to discover: the higgs boson machine learning challenge. $U R L$ http://higgsml. lal. in2p3. fr/documentation, page 9, 2014.

[2] Claire Adam-Bourdarios, Glen Cowan, Cécile Germain, Isabelle Guyon, Balázs Kégl, and David Rousseau. The higgs boson machine learning challenge. In NIPS 2014 Workshop on High-energy Physics and Machine Learning, pages 1955, 2015.

[3] Dzmitry Bahdanau, Kyunghyun Cho, and Yoshua Bengio. Neural machine translation by jointly learning to align and translate. arXiv preprint arXiv: 1409.0473, 2014.

[4] Gérard Biau, Benoît Cadre, and Laurent Rouvière. Accelerated gradient boosting. Machine Learning, pages 1-22, 2018.

[5] Tianqi Chen and Carlos Guestrin. Xgboost: A scalable tree boosting system. In Proceedings of the 22nd acm sigkdd international conference on knowledge discovery and data mining, pages 785-794. ACM, 2016.

[6] Tianqi Chen and Tong He. Higgs boson discovery with boosted trees. In NIPS 2014 workshop on high-energy physics and machine learning, pages 69-80, 2015.

[7] Ting Chen, Lu-An Tang, Yizhou Sun, Zhengzhang Chen, and Kai Zhang. Entity embedding-based anomaly detection for heterogeneous categorical events. In Proceedings of International Joint Conferences on Artifical Intelligence (IJCAI), pages 1396-1403, 2016.

[8] Li Deng. The mnist database of handwritten digit images for machine learning research [best of the web]. IEEE Signal Processing Magazine, 29(6):141-142, 2012.

[9] Jacob Devlin, Ming-Wei Chang, Kenton Lee, and Kristina Toutanova. Bert: Pre-training of deep bidirectional transformers for language understanding. arXiv preprint arXiv:1810.04805, 2018.

[10] Jeff Donahue, Yangqing Jia, Oriol Vinyals, Judy Hoffman, Ning Zhang, Eric Tzeng, and Trevor Darrell. Decaf: A deep convolutional activation feature for generic visual recognition. In International conference on machine learning, pages 647-655, 2014.

[11] Dheeru Dua and Efi Karra Taniskidou. UCI machine learning repository, 2017.

[12] R.A. Fisher. Iris data set, 1936.

[13] Andrew Fogg. Anthony goldbloom gives you the secret to winning kaggle competitions, 2016.

[14] PARVUS Forina, M. et al. Wine data set, 1991.

[15] Cheng Guo and Felix Berkhahn. Entity embeddings of categorical variables. arXiv preprint arXiv:1604.06737, 2016.

[16] Kaiming He, Xiangyu Zhang, Shaoqing Ren, and Jian Sun. Deep residual learning for image recognition. In Proceed- ings of the IEEE conference on computer vision and pattern recognition, pages 770-778, 2016.

[17] Jie Hu, Li Shen, and Gang Sun. Squeeze-and-excitation networks. In Proceedings of the IEEE conference on computer vision and pattern recognition, pages 7132-7141, 2018.

[18] Armand Joulin, Edouard Grave, Piotr Bojanowski, and Tomas Mikolov. Bag of tricks for efficient text classification. arXiv preprint arXiv:1607.01759, 2016.

[19] Guolin Ke, Qi Meng, Thomas Finley, Taifeng Wang, Wei Chen, Weidong Ma, Qiwei Ye, and Tie-Yan Liu. Lightgbm: A highly efficient gradient boosting decision tree. In Advances in Neural Information Processing Systems, pages 3146-3154, 2017.

[20] Ronny Kohavi and Barry Becker. Adult data set, 1996.

[21] Hoang Thanh Lam, Tran Ngoc Minh, Mathieu Sinn, Beat Buesser, and Martin Wistuba. Neural feature learning from relational database. arXiv preprint arXiv:1801.05372, 2018.

[22] Chenxi Liu, Barret Zoph, Maxim Neumann, Jonathon Shlens, Wei Hua, Li-Jia Li, Li Fei-Fei, Alan Yuille, Jonathan Huang, and Kevin Murphy. Progressive neural architecture search. In Proceedings of the European Conference on Computer Vision (ECCV), pages 19-34, 2018.

[23] Tomas Mikolov, Ilya Sutskever, Kai Chen, Greg S Corrado, and Jeff Dean. Distributed representations of words and phrases and their compositionality. In Advances in neural information processing systems, pages 3111-3119, 2013.

[24] Sinno Jialin Pan and Qiang Yang. A survey on transfer learning. IEEE Transactions on knowledge and data engineering, 22(10):1345-1359, 2010.

[25] Jeffrey Pennington, Richard Socher, and Christopher Manning. Glove: Global vectors for word representation. In Proceedings of the 2014 conference on empirical methods in natural language processing (EMNLP), pages 1532-1543, 2014.

[26] Matthew E Peters, Mark Neumann, Mohit Iyyer, Matt Gardner, Christopher Clark, Kenton Lee, and Luke Zettlemoyer. Deep contextualized word representations. arXiv preprint arXiv:1802.05365, 2018.

[27] Liudmila Prokhorenkova, Gleb Gusev, Aleksandr Vorobev, Anna Veronika Dorogush, and Andrey Gulin. Catboost: unbiased boosting with categorical features. In Advances in Neural Information Processing Systems, pages 6639-6649, 2018.

[28] Alec Radford, Karthik Narasimhan, Tim Salimans, and Ilya Sutskever. Improving language understanding by generative pre-training. URL https://s3us-west-2. amazonaws. com/openai-assets/researchcovers/languageunsupervised/language understanding paper. pdf, 2018.

[29] Olga Russakovsky, Jia Deng, Hao Su, Jonathan Krause, Sanjeev Satheesh, Sean Ma, Zhiheng Huang, Andrej Karpathy, Aditya Khosla, Michael Bernstein, Alexander C. Berg, and Li Fei-Fei. ImageNet LargeScale VisualRecognition Challenge. International Journal of Computer Vision (IJCV), 115(3):211-252, 2015.

[30] Ali Sharif Razavian, Hossein Azizpour, Josephine Sullivan, and Stefan Carlsson. Cnn features off-the-shelf: an astound- 
ing baseline for recognition. In Proceedings of the IEEE conference on computer vision and pattern recognition workshops, pages 806-813, 2014.

[31] Baohua Sun, Daniel Liu, Leo Yu, Jay Li, Helen Liu, Wenhan Zhang, and Terry Torng. Mram co-designed processingin-memory cnn accelerator for mobile and iot applications. arXiv preprint arXiv:1811.12179, 2018.

[32] Baohua Sun, Lin Yang, Catherine Chi, Wenhan Zhang, and Michael Lin. Squared english word: A method of generating glyph to use super characters for sentiment analysis. arXiv preprint arXiv:1902.02160, 2019.

[33] Baohua Sun, Lin Yang, Patrick Dong, Wenhan Zhang, Jason Dong, and Charles Young. Super characters: A conversion from sentiment classification to image classification. In Proceedings of the 9th Workshop on Computational Approaches to Subjectivity, Sentiment and Social Media Analysis, pages 309-315, 2018.

[34] Baohua Sun, Lin Yang, Patrick Dong, Wenhan Zhang, Jason Dong, and Charles Young. Ultra power-efficient cnn domain specific accelerator with 9.3 tops/watt for mobile and embedded applications. In Proceedings of the IEEE Conference on Computer Vision and Pattern Recognition Workshops, pages 1677-1685, 2018.

[35] Rachel Thomas. An introduction to deep learning for tabular data, 2018.

[36] Ashish Vaswani, Noam Shazeer, Niki Parmar, Jakob Uszkoreit, Llion Jones, Aidan N Gomez, Łukasz Kaiser, and Illia Polosukhin. Attention is all you need. In Advances in Neural Information Processing Systems, pages 5998-6008, 2017.

[37] William Vorhies. Has deep learning made traditional machine learning irrelevant?, 2016.

[38] Jason Yosinski, Jeff Clune, Yoshua Bengio, and Hod Lipson. How transferable are features in deep neural networks? In Advances in neural information processing systems, pages 3320-3328, 2014.

[39] Xingcheng Zhang, Zhizhong Li, Chen Change Loy, and Dahua Lin. Polynet: A pursuit of structural diversity in very deep networks. In Proceedings of the IEEE Conference on Computer Vision and Pattern Recognition, pages 718-726, 2017.

[40] Barret Zoph, Vijay Vasudevan, Jonathon Shlens, and Quoc V Le. Learning transferable architectures for scalable image recognition. In Proceedings of the IEEE conference on computer vision and pattern recognition, pages 8697-8710, 2018. 